\title{
Las políticas públicas a debate: aportes desde la antropología y la sociología de la traducción
}

\author{
Public policy under debate: contributions \\ from anthropology and sociology of translation
}

\author{
Sandra Patricia Martínez B. ${ }^{*}$ \\ Universidad del Valle, Colombia
}

DOI: $10.22380 / 2539472 X .2023$

\begin{abstract}
RESUMEN
En este artículo nos proponemos ponderar las contribuciones de la aproximación antropológica y de la sociología de la traducción al estudio de las políticas públicas. A partir de la revisión de la literatura publicada en América Latina en las últimas dos décadas pudimos constatar que, si bien cada una de estas perspectivas se enmarca en referentes conceptuales y estrategias metodológicas particulares, ambas cuestionan la visión de las políticas como un instrumento técnico y racional para resolver problemas. Asimismo, al considerar el entramado de entidades humanas y no humanas, instituciones, conocimientos, valores, prácticas y artefactos que intervienen en la construcción de las políticas públicas, estos enfoques permiten reconocer el carácter disputado e inacabado de dichas políticas, al tiempo que complejizan la mirada del "éxito" o "fracaso" de los programas como el producto temporal de asociaciones inestables.
\end{abstract}

Palabras clave: políticas públicas, antropología de las políticas públicas, sociología de la traducción, teoría del actor-red.

\begin{abstract}
In this article we propose to consider the contributions of the anthropological approach and the sociology of translation to the study of public policy. Based on a review of the literature published in Latin America over the last two decades, we have found that although each of these perspectives is framed by particular conceptual references and methodological strategies, both question the view of policy as a technical and rational instrument to solve problems. Likewise, by considering the network of human and non-human entities, institutions, knowledge, values, practices and artifacts involved in the construction of public policies, these approaches allow us to recognize the contested and unfinished nature of such policies, while at the same time problematizing the view of the "success" or "failure" of programs as the temporary product of unstable associations.
\end{abstract}

Keywords: public policy, anthropology of public policy, sociology of translation, actornetwork theory.

* $\quad$ sandra.p.martinez@correounivalle.edu.co / https://orcid.org/0000-0002-4411-1349 


\section{Introducción}

Este artículo tiene como propósito justipreciar los aportes de la aproximación antropológica y de la sociología de la traducción al estudio de las políticas públicas $^{1}$. En lo que toca al primero de estos enfoques, por lo menos desde hace tres décadas, el análisis ha adquirido un creciente interés entre los antropólogos políticos, quizá porque plantea cuestiones centrales a su ámbito disciplinar, tales como los modos de acceso y ejercicio del poder, los sistemas políticos y las formas de gobierno, las instituciones y normas sociales, el estado ${ }^{2}$ y sus mecanismos de legitimación, el conocimiento y la autoridad, el lenguaje y el discurso, entre otras. Al incursionar en el campo de las políticas públicas, los antropólogos se han abierto paso en el estudio de un fenómeno cardinal en las sociedades contemporáneas, que funciona como un termómetro de las transformaciones en las modalidades de ejercicio de gobierno y de la manera en que estas inciden en la configuración de las relaciones entre el individuo y la sociedad. Es así como, en el propósito de recuperar la perspectiva de los actores, propia de los abordajes etnográficos, los antropólogos han insistido en la necesidad de trascender la dimensión evaluativa de los resultados de las políticas, para tratar de comprender, en cambio, lo que hacen y piensan tanto quienes las diseñan e implementan como los sujetos a quienes van dirigidas (Shore 2010).

Con relación a la sociología de la traducción, aunque inicialmente sus contribuciones se situaron en el campo de los estudios sociales de la ciencia y la tecnología (ESCT), es preciso señalar que, en los últimos años, esta perspectiva de análisis ha sido aplicada en ámbitos de conocimiento tan diversos como la salud pública, la educación, la psicología, la economía, el urbanismo, las ciencias de la comunicación, la antropología virtual y los estudios rurales y ambientales, por solo mencionar algunos. Si bien el examen de las políticas públicas desde este segundo enfoque es más reciente, estamos convencidos de su potencial analítico para dilucidar el complejo entramado de actores humanos y no humanos,

1 Aunque la expresión teoría del actor red, y su acrónimo en inglés, ANT, han gozado de una mayor aceptación en el mundo anglófono que la expresión sociología de la traducción, en este trabajo optamos por el uso de este último término, en el que el mismo Latour (2008) reconoce un mayor grado de precisión. Dicho enfoque es comúnmente asociado al campo de los estudios sociales de la ciencia y la tecnología (ESCT), pero ha trascendido hacia otros ámbitos disciplinares y objetos de estudio, como el análisis de las políticas públicas, abordado en este artículo.

2 Nos suscribimos a la postura teórica adoptada por la antropología del estado, en la que se ha optado por no escribir las palabras estado y gobierno con mayúscula inicial como una manera de significar una ruptura con los enfoques convencionales que ven estos fenómenos como entidades monolíticas y todopoderosas situadas por encima de la sociedad. 
intereses y programas de acción que intervienen en la construcción de las políticas.

Después de hacer una breve revisión de la utilería conceptual y del encuadre metodológico que forman parte de estas dos aproximaciones, señalamos sus puntos de encuentro en torno a la lectura que proponen de las políticas públicas. Seguidamente, presentamos un balance preliminar de las investigaciones realizadas en América Latina que se han sustentado en dichos enfoques para iluminar el estudio de las políticas; con este propósito nos apoyamos en la búsqueda de artículos en revistas científicas registradas en las principales bases de datos de la región, así como en la consulta de otras fuentes bibliográficas que se han publicado sobre el tema. Cerramos el texto con algunas reflexiones sobre los aportes y desafíos de encarar dicho fenómeno desde estas grillas analíticas.

\section{El estudio de las políticas públicas: miradas diversas}

En el ámbito de los estudios de las políticas públicas pueden identificarse dos aproximaciones claramente diferenciadas. Las décadas de los años sesenta y setenta estuvieron dominadas por una visión instrumentalista de las políticas, en la que eran concebidas como un instrumento técnico y racional para resolver problemas. Desde este enfoque, aún vigente en ciertos ámbitos académicos e institucionales, las políticas son representadas como un proceso lineal, lógico y ordenado que empieza con la identificación de un problema, continúa con la formulación de una solución y termina con la implementación y evaluación de unos programas. Esta mirada supone una doble lógica de diagnóstico y prescripción, según la cual los tomadores de decisiones definen unos medios y unos fines, evalúan las distintas posibilidades existentes y, orientados por una racionalidad de costo-beneficio, eligen la mejor alternativa. Todo ello bajo el supuesto de que la información y el conocimiento garantizan no solo la formulación e implementación de políticas eficaces, sino también una representación objetiva y neutral de la realidad a intervenir (Shore 2010; Shore y Wright 1997; Wedel et al. 2005).

A partir de los años ochenta, algunos autores comienzan a refutar esta consideración de las políticas públicas como procesos lineales, que se construyen de arriba abajo siguiendo una serie de pasos que inician con su formulación en los altos niveles administrativos y luego descienden hacia los rangos medios hasta llegar a los burócratas de la calle (Lipsky [1980] 1999), quienes finalmente 
se hacen cargo de su implementación (Shore 2010). Los críticos de esta perspectiva plantean la necesidad de reconocer el carácter ambiguo y disputado de las políticas, así como de adoptar una perspectiva orientada al actor que permita dar cuenta de la manera en que los individuos y las redes que estos constituyen coproducen los mundos sociales en los que se desenvuelven. Desde esta aproximación, los sujetos de las políticas no aparecen como meros receptores de unos proyectos ideados “desde arriba”, sino que despliegan distintas estrategias y recursos frente a las intervenciones planeadas por agencias públicas o privadas, al tiempo que las incorporan en sus mundos de vida y les otorgan significados disímiles (Long [2001] 2007). Se trata de una mirada crítica que toma la política pública como un objeto de análisis en sí mismo y no como un hecho que pueda darse por sentado.

Uno de los aspectos que ha sido objeto de escrutinio por parte de los autores que se adscriben a este punto de vista son las narrativas de las políticas que, al revestirse del lenguaje objetivo, neutral y legal-racional de la ciencia, aparecen como instrumentos dirigidos a promover la eficiencia y la efectividad, por lo que enmascaran, de esta manera, los verdaderos fines políticos que persiguen. Al representar cuestiones políticas como la pobreza, el acceso a la tierra y los recursos, el trabajo y los ingresos como problemas de orden técnico, las políticas tienen un poderoso efecto conceptual e ideológico de despolitización de la vida social. Esta apelación a un lenguaje científico y aparentemente neutral no tiene otra función distinta a la de silenciar la oposición, cuyos argumentos en contra de las decisiones tomadas suelen ser descalificados como "irracionales" o "regresivos”. En tal sentido, las políticas públicas cumplen una función claramente legitimadora, que busca apuntalar la autoridad de quienes se encuentran en posiciones de autoridad (Escobar [1995] 2007; Ferguson 1994; Li 2007; Scott 1998; Shore 2010; Shore y Wright 1997; Wedel et al. 2005).

Afín a esta segunda aproximación, la antropología de las políticas públicas se propone develar las constelaciones de actores, operaciones e intereses que configuran las decisiones, así como su implementación y los efectos que estas traen consigo. Antes que interesarse en establecer una definición abstracta de "la política”, el antropólogo se propone auscultar qué hace la gente en nombre de la política (Wedel et al. 2005), cuáles son los marcos de referencia de quienes las diseñan e implementan, y cuál es el carácter de las relaciones que establecen con sus destinatarios. El conocimiento de las diversas experiencias e interpretaciones que los pobladores hacen de estas políticas ocupa un lugar igualmente importante dentro de este enfoque (Shore 2010). En diálogo con otras aproximaciones críticas al estado y a las formas de ejercicio del gobierno, la perspectiva antropológica de las políticas públicas cuestiona las habituales antinomias que 
han cartografiado el estudio del poder político y que han establecido una frontera precisa entre el estado y la sociedad civil, lo público y lo privado, lo legal y lo ilegal, lo formal y lo informal, el centro y los márgenes, entre otras.

Además del cuestionamiento a dichas dicotomías, este enfoque parte de la constatación empírica del divorcio existente entre los objetivos fijados al inicio de la intervención y los resultados efectivamente alcanzados. Al decir de Rose y Miller (1992), “el gobierno es una operación congénitamente fallida” (190). En su proceso de aplicación práctica, las políticas tropiezan con una serie de obstáculos que escapan a la lógica programática de los planificadores (falta de recursos financieros, rivalidades profesionales, ausencia de estadísticas confiables, sistemas de comunicación ineficaces, líneas de mando difusas), por no mencionar aquellos derivados de las diversas lecturas e interpretaciones, cuando no de la resistencia por parte de los sujetos a quienes van dirigidas. Esto es así porque, lejos de ser instrumentos técnicos pretendidamente neutrales ideados para la resolución de problemas, las políticas públicas están inmersas en las luchas por el poder y la dominación que se despliegan entre actores diferencialmente situados en los campos en los que ellas se insertan (Li 2007; Shore 2010; Shore y Wright 1997; Wedel et al. 2005).

A finales de la década de los noventa, Cris Shore y Susan Wright (1997) sentaron las bases de una agenda de investigación en el campo de la antropología de las políticas públicas. Para ello, los autores organizaron el debate en torno a tres posibles maneras de entender dichas políticas: como lenguaje de poder, como agente cultural y como tecnología política. Desde la primera de estas acepciones, se considera que el lenguaje de las políticas tiene la capacidad de otorgar autoridad institucional a unos discursos por encima de otros. Al respecto, Raymond Apthorpe (1997) ha planteado que el lenguaje en el que se expresan las políticas funciona como un mecanismo de poder que tiene la finalidad de persuadir antes que de informar. De ahí que sea importante pensar en la política escrita como un género, un estilo de expresión que es seleccionado y perseguido. Los lenguajes y estilos de la política son, entonces, un reflejo de los valores culturales y de los sistemas morales de una sociedad.

En lo que tiene que ver con la consideración de las políticas como agentes culturales, se destaca el papel que desempeñan en el control y regulación de poblaciones heterogéneas, a través de sistemas de clasificación que intentan homogeneizar tal diversidad. Al clasificar a la población, las políticas tienen un activo rol en la creación de nuevas categorías de individuos, quienes son definidos de acuerdo con diferentes criterios de clase, género, pertenencia étnica, edad, profesión, ingresos, entre muchos otros. De este modo, se terminan imponiendo tipos ideales de ciudadanía, que son apropiados de diversas maneras por los 
sujetos. Pero esta imposición no es de ningún modo coercitiva, sino que consiste en la creación de un campo de elecciones razonables de lo que se debe y no se debe hacer, a partir del cual los individuos normalizan determinados patrones de acción y comportamiento (Wedel et al. 2005). Este es, precisamente, uno de los rasgos centrales de las modalidades neoliberales de gobierno, en las que la autonomía personal entra a formar parte de los mecanismos de operación del poder político, al lado de métodos más sutiles de intervención basados en ideas como la libertad, la empresa y el mercado (Dean 2002; Rose 1996; Rose y Miller 1992).

Por su parte, la consideración de las políticas como tecnologías políticas ve en ellas formas de poder que obran sobre el sentido individual del ser, de tal suerte que el foco de interés de quienes se adhieren a esta mirada se centra en cómo los individuos adoptan e internalizan los nuevos patrones de conducta configurados por las democracias liberales avanzadas (Shore y Wright 1997). Los fundamentos de esta proposición hunden sus raíces en la noción de gubernamentalidad, a partir de la que Foucault (2006) da cuenta de la aparición del problema de la población como dato, como fin e instrumento de las técnicas de gobierno y como campo de intervención. Se trata de una nueva racionalidad gubernamental que cifra su eficacia, ya no en mecanismos coercitivos, sino en la libertad como tecnología de conducción de la conducta dirigida a la autorregulación de los individuos (Rose 1996; Shore y Wright 1997).

En este punto, es importante referirnos al cuestionamiento que algunos autores les han hecho a estos dos enfoques, el instrumental y el crítico. Mosse (2005), por ejemplo, considera que ambas aproximaciones obstaculizan la comprensión de la relación existente entre las políticas y la práctica, por cuanto desconocen el complejo entramado de actores, intereses y cursos de acción implicados en su desarrollo. La primera, al establecer de manera ingenua una relación demasiado mecánica entre el diseño y la aplicación de las políticas. La segunda, al calificarlas como dispositivos de dominación y control que reproducen las jerarquías de conocimiento, silencian las voces de sus destinatarios y ocultan sus verdaderos fines políticos de regulación social y autoridad burocrática tras el velo de un lenguaje científico que se pretende neutral y racional. En coincidencia con la perspectiva centrada en el actor propuesta por Long ([2001] 2007) para el estudio de la intervención planeada, Mosse (2005) plantea la necesidad de restituir la capacidad de agencia de los actores implicados en las diversas arenas del desarrollo que, a su juicio, permitirá superar la mirada de los destinarios de las políticas como víctimas engañadas, y de las instituciones y sus profesionales como actores todopoderosos. 
A partir de su experiencia como consultor en un proyecto de desarrollo rural ejecutado en la India, Mosse (2005) se propone abrir la caja negra ${ }^{3}$ de las prácticas y su relación con las prescripciones de política, un aspecto poco atendido en la literatura sobre el tema o cuyos desajustes han sido explicados en términos de una brecha entre la teoría y la práctica, bajo la premisa de que, a mejores modelos, mayores posibilidades de éxito en la implementación de las políticas. Inspirado en la perspectiva orientada al actor, así como en la sociología de la traducción, el autor sostiene que la función de las políticas públicas, entendidas como modelos y diseños de proyectos, no consiste en orientar las prácticas de desarrollo, sino en movilizar y ganar el apoyo político necesario para legitimar las intervenciones. Así, aunque el lenguaje en el que se expresan las políticas es técnico, sus fines son políticos, en el sentido de que buscan convocar a distintos actores (gobiernos, organizaciones no gubernamentales, agencias donantes, consultores) con agendas e intereses divergentes en torno a la localización de recursos en proyectos particulares. Esto significa que el papel de las políticas no reside precisamente en orientar la acción, sino más bien en lograr alianzas, coaliciones y consensos entre los diferentes actores implicados, sin quienes estos proyectos no llegarían a hacerse realidad.

Detenernos en esta última proposición nos conduce a los aportes de la sociología de la traducción, una de cuyas figuras más sobresalientes es Bruno Latour. Su teoría del actor-red (TAR) plantea que el quehacer del sociólogo consiste en el rastreo de las asociaciones, a partir de la adopción del principio metodológico de "seguir a los actores" para comprender la forma en que estos definen sus realidades. Para Latour (2008), los actores se hallan insertos en redes más amplias en las que cada participante está en capacidad de hacer que los otros hagan algo, lo que desencadena desplazamientos, eventos inesperados o nuevas interpretaciones.

Esta capacidad de hacer que otros hagan algo pasa por el trabajo de traducción realizado por algunos agentes, quienes además de alinear los intereses de otros actores humanos en torno a un proyecto determinado, articulan entidades no humanas a la red. Sin embargo, cabe precisar que, siendo consecuentes con

3 Para Latour, la expresión caja negra tiene un sentido más amplio. En Ciencia en acción (1992), el autor cuestiona la consideración modernista del conocimiento científico como un producto terminado, del cual apenas es posible conocer los datos de entrada y de salida, para proponer, en cambio, el estudio de los hechos tecnocientíficos en tiempo real, es decir, mientras se están produciendo: "Estudiamos la ciencia en acción y no la ciencia y la tecnología ya elaboradas; para ello o bien llegamos antes de que los hechos y las máquinas se conviertan en cajas negras, o bien estudiamos las controversias que las vuelven a abrir" (139). De este modo, al abrir las cajas negras se hace visible el conjunto de hechos y aparatos que habían quedado ocultos mediante la operación de simplificación. 
el principio de simetría propugnado por este enfoque analítico, los teóricos de la TAR plantean que la capacidad de agencia no es una cualidad exclusiva de las personas, sino que también es ostentada por los objetos, dispositivos y demás materialidades que, lejos de ser simples intermediarios que transportan significados sin transformarlos, actúan como mediadores que modifican, traducen o alteran dichos significados (Latour 2008).

La noción de traducción es, pues, central a la propuesta de hacer una sociología de las asociaciones planteada por esta vertiente analítica. El proceso de traducción abarca el conjunto de negociaciones, alianzas, acciones de persuasión o coercitivas que un agente ejerce para lograr la adhesión de otros actores a sus propios intereses (Callon 1995; Latour 1992). Este proceso implica tanto el desplazamiento de metas e intereses, como de materiales, recursos e información, que dejan de ser entidades pasivas, para incorporarse a entramados móviles, plenos de significado y desencadenantes de acciones. Así, llevar a la realidad un proyecto no es algo que dependa de la coherencia de su diseño inicial, sino de la habilidad de algunos actores para lograr el consenso en torno a este.

Los teóricos de la TAR (Callon 1995; Latour 1996; Law 1998) distinguen cuatro fases constitutivas del proceso de traducción: 1) el momento de la problematización, en el que los actores construyen sus propias hipótesis acerca de la identidad, la conducta y las metas de otros actores (sean humanos o no) y, a partir de ello, definen puntos de paso obligados para forzarlos a moverse por canales particulares; 2) el interesamiento, que encierra el conjunto de acciones desplegadas por un actor a fin de imponer y estabilizar la identidad que les ha atribuido a otros actores; 3) el enrolamiento, esto es, el conjunto de negociaciones, acciones de fuerza, concesiones y consensos a través de los cuales se definen y asignan determinados roles a los actores; 4) la movilización de actores que, a través de la designación de portavoces, busca concitar el interés de otros agentes en torno a un proyecto determinado.

Como resultado de esta cadena de operaciones de traducción, tenemos la construcción de una compleja red de relaciones, cuyas características han sido descritas con el término actor-red.

En palabras de Callon (1998): “Un actor-red es, simultáneamente, un actor cuya actividad consiste en entrelazar elementos heterogéneos y una red que es capaz de redefinir y transformar aquello de lo que está hecha” (156). Con esta definición, el autor pretende significar al actor-red como la acción misma que articula temporalmente entidades heterogéneas, sean humanas o no. En este sentido, las redes están constituidas tanto por entidades naturales como sociales que, lejos de ser estables, se encuentran en un continuo proceso de redefinición de sus identidades y de sus relaciones mutuas. Esta dinámica de asociación de 
componentes heterogéneos es posible gracias a dos operaciones: simplificación y yuxtaposición. La primera consiste en la reducción que los actores hacen de la realidad a partir de la definición de unas cuantas entidades a las que les son atribuidas ciertas características. De lo anterior se infiere que tales definiciones siempre se hallan en competencia con otras, por lo que su aseguramiento requiere de un continuo trabajo de estabilización. Pero estas entidades no existen de manera aislada, sino que se encuentran ligadas a otras que hacen parte de la red. Es aquí donde interviene la yuxtaposición que, al poner en relación a las entidades simplificadas, las sitúa en un contexto en el cual adquieren significado.

En esta apretada síntesis del enfoque de la TAR se vislumbra el valor heurístico de la sociología de la traducción para el estudio de las políticas públicas que, mediante el principio metodológico de seguir a los actores, hace posible reconstruir la cadena de asociaciones, intereses, acciones, recursos y desplazamientos de significados que estos movilizan para adherir a otros agentes (humanos y no humanos) a su causa, en este caso, una política pública en particular. Al revelar el carácter inestable de las asociaciones, identidades e interpretaciones alcanzadas en el decurso de los procesos de traducción, esta perspectiva analítica permite, asimismo, comprender los pasos en falso, las intenciones frustradas o las tentativas fallidas que en muchas ocasiones impiden que un proyecto llegue a existir, lo que derriba las lecturas convencionales de las políticas que sitúan su "fracaso" en el desajuste entre los diseños y la realidad.

Llegados a este punto, podemos establecer un claro paralelo entre la aproximación antropológica a las políticas públicas y la TAR. Desde el primer enfoque, estas políticas son entendidas como principios organizadores respecto a los cuales la gente estructura sus realidades, a través de la creación de vínculos entre agentes, instituciones, tecnologías y discursos que buscan alinear esos diversos elementos (Shore y Wright 2011). Desde la TAR, podríamos entender las políticas públicas como el resultado de la asociación heterogénea de actores, instituciones, conocimientos, prácticas, valores, artefactos, entre otros, que son reunidos, durante un periodo de tiempo, en el mismo lugar ${ }^{4}$, en torno a su construcción (Greenhalgh 2008). De este modo, podemos afirmar que las políticas públicas funcionan a la manera de un actor-red que, según Latour (2008), no es lugares, por cuanto estos son articulados a la red a través del trabajo de contextualización realizado por los distintos agentes implicados. Al respecto, Grau, Íñiguez y Subirats (2010) señalan que: "Desde esta perspectiva, en definitiva, la distinción entre contexto y contenido se vuelve inservible, puesto que uno y otro no son discernibles entre sí. Y permite concebir, en el análisis de políticas públicas, cómo un acontecimiento pasado, lejano o con protagonistas ausentes puede seguir presente, si se consigue traducirlo, desplazarlo, inscribirlo en otros elementos, a poder ser, lo más móviles y duraderos posible" (78). 
otra cosa que "el blanco móvil de una enorme cantidad de entidades que convergen hacia él” (73).

A continuación presentamos un balance, que en absoluto pretende ser exhaustivo, de las investigaciones efectuadas en América Latina en las que se han adoptado las perspectivas socioantropológica y de la sociología de la traducción para abordar el fenómeno de las políticas públicas.

\section{Aproximaciones socioantropológicas a las políticas públicas}

Dejando de lado cualquier pretensión de abarcar la totalidad de la literatura sobre el tema, podemos decir que los aportes al estudio de este fenómeno en América Latina por parte de los antropólogos o de otros científicos sociales que recurren a métodos etnográficos se han centrado básicamente en las líneas de análisis que se esbozan a continuación. En un primer énfasis temático, gran parte de estas investigaciones se ha dirigido al examen de los efectos que las políticas públicas producen en la vida de las poblaciones a las cuales se dirigen. De una parte, estos trabajos abordan la manera en que los actores vinculados al mundo de las políticas recortan ciertos segmentos de la realidad para construirlos como áreas problemáticas que deben ser objeto de intervención (Assusa 2014; Barbato 2005; Chao 2019; Fagundez y González 2018). De otra parte, se ocupan de la incidencia que dichos dispositivos estatales tienen en la construcción de nuevas subjetividades entre sus beneficiarios, quienes adoptan las identidades que les son atribuidas por instituciones y funcionarios para hacerse merecedores de los servicios dispensados por las agencias estatales (Aparicio 2005; Gracia 2015; Grinberg 2008; Jardim 2016; Ortiz 2017).

Ahora bien, no todos los sujetos etnográficos indagados muestran tal nivel de conformidad, pues como lo han evidenciado algunos autores, los destinatarios de las políticas son, a la vez, objetos y agentes de ellas, por cuanto elaboran sus propias interpretaciones y traducciones de dichos modelos de acuerdo con sus intereses, valores y necesidades (Agudo 2009, 2011; Dapuez 2016; Hornes 2014; Isacovich 2013; S. Martínez 2017). Cabe pensar, sin embargo, que las formas de intervención estatal también se ven afectadas por los contextos en los que operan, en tanto que, en ocasiones, adoptan las formas locales de organización a fin de favorecer el desarrollo de sus iniciativas (Dallorso 2012; Lozano 2016; Llanes 2009). 
Un segundo énfasis temático aborda el estudio de las interacciones entre los funcionarios y operadores de las políticas públicas y las poblaciones asistidas a partir del examen de las prácticas y representaciones desplegadas por unos y otros en esta interacción. Para algunos de los autores que se inscriben en esta línea, la aplicación de la ley y de los dispositivos institucionales es el resultado del “regateo” y la negociación entre estos actores (S. Martínez 2019; Nuijten 2003; Recalde 2019), mientras que para otros, en dichos encuentros se reproducen y actualizan las jerarquizaciones sociales y, con ellas, los parámetros de inclusión y exclusión de los ciudadanos en las políticas estatales (Diehl y Langdon 2015; Franco 2016; Restrepo 2010; Salaini y Jardim 2015; Saldi 2016; Wanderley 2009). Ahora bien, los modelos institucionales de intervención devienen objetos de cuestionamiento tanto por parte de sus beneficiarios (Jaramillo 2006, 2008; Mancini 2016; Mariano 2013; Osorio 2014), como de los mismos agentes a cargo de su implementación.

En este complejo campo de interacciones, emergen diversas respuestas por parte de sus destinatarios, que van desde la sumisión a la autoridad estatal o el desistimiento de las solicitudes (Auyero 2013), pasando por la realización de las performances esperadas por los funcionarios con el fin de asegurar su inclusión o permanencia en los programas (Herron 2003; Hornes y Salerno 2019; Jabs 2013; Medan 2018), hasta la construcción de nuevas identidades al margen de las previsiones de estos últimos (Jaramillo 2006; F. Martínez Ramírez 2019; Osorio 2011). Los funcionarios, por su parte, elaboran toda suerte de taxonomías, que definen y categorizan a los usuarios de las políticas de acuerdo con la dimensión de sus carencias. Así, quienes no logran demostrar su calidad de "necesitados” son calificados como “aprovechados” (Mariano 2013), sospechosos e, incluso, peligrosos (Recalde 2019).

El papel de las burocracias en el diseño e implementación de las políticas públicas constituye el eje de una tercera vertiente de análisis. En este grupo se sitúan los estudios interesados en develar los valores y hábitos que los agentes estatales intentan inculcar en las poblaciones intervenidas (Assusa 2014; Gutiérrez y Assusa 2015), aquellos que examinan las limitaciones presupuestales y organizacionales que estos enfrentan en el desarrollo de su trabajo (Jaramillo 2008; Salej, Ardila y Bragato 2016), así como los que abordan los amplios márgenes de discrecionalidad de los funcionarios a la hora de tomar decisiones sobre la asignación de bienes y servicios públicos (Buchely 2015; Perelmiter 2016). En esta vertiente pueden incluirse también las investigaciones que se ocupan del rol que los saberes expertos desempeñan tanto en la configuración de diferentes formas de intervención social y modelos de gobierno (Chávez y Piquinela 2017; Franco 
2016; García 2016; Hornes 2020; Salaini y Jardim 2015) como en la evaluación de los programas sociales (Agudo 2009).

\section{Las políticas públicas vistas a través de la sociología de la traducción}

Las nociones y principios que hacen parte de la sociología de la traducción han sido incorporados al análisis de las políticas públicas que, vistas desde esta perspectiva, adquieren un nuevo sentido que se aleja de los enfoques normativos convencionales. Acogiendo los planteamientos de la TAR, un creciente número de autores ha conceptualizado las políticas públicas como un ensamblaje de elementos heterogéneos, tanto sociales como técnicos, semánticos o materiales, que logran ser enrolados y movilizados durante algún tiempo, ya sea para apoyar y estabilizar ciertos modelos de política, o para legitimar la definición oficial de los problemas y de los sujetos de intervención estatal. Este enfoque ha sido fructífero para dar cuenta de la complejidad de un amplio rango de políticas en áreas tan diversas como el manejo del riego (Rap 2008), el ordenamiento territorial (Cabrera 2011), la pobreza (Corrêa y Spink 2014; Ramos 2016), el transporte público (Ureta 2014, 2015), la inclusión social de personas en situación de calle (Chávez y Riet 2015; Fagundez 2018), la educación artística (Jaramillo 2015), las políticas de juventud (Faustino y Hüning 2015), la salud pública (E. Martínez 2016), la atención y restitución de las víctimas del conflicto armado (Dávila 2017; Mora 2016), los sistemas educativos (Amantino y Arimatéia 2017; Da Silva y López 2014; Ramos 2018), la lucha contra la corrupción (Arenas y Alzate 2019) y el empoderamiento (Castillo, Winkler y Berroeta 2019).

Otras investigaciones adoptan algunas nociones de este corpus teórico para aplicarlas a estudios de caso particulares. Es el caso de los trabajos de D’Alessandro y González (2014), quienes se apoyan en las nociones de redes sociotécnicas y de controversias para describir las tensiones generadas por la implementación de un programa de distribución de semillas de maíz mejorado en los Altos de Chiapas, y de la investigación de Kerr, Santos y Gastalho (2015) sobre la política de internación obligatoria para los usuarios de drogas en Río de Janeiro. El concepto de redes sociotécnicas ha sido utilizado, además, por otros autores como Salete et al. (2015) para examinar el Programa de Erradicación del Trabajo Infantil en Brasil y por Schöngut (2017) en su indagación sobre los programas de protección de menores en Chile. 
De igual modo, la noción de traducción ha mostrado ser productiva en el análisis de intervenciones gubernamentales específicas. Es así como algunos autores recurren a ella para explicar la manera en que los programas de política social se hacen reales gracias al trabajo de generación y traducción de intereses desplegado por los brokers. Este rol es ejercido por distintos actores, como los proveedores del servicio de salud, los funcionarios y las lideresas comunitarias del Programa Oportunidades en México (Agudo 2009; 2011) y los “operadores de cercanía” de la política Uruguay Crece Contigo (Chávez, Hotou y Piquinela 2016). Por su parte, Chávez y Piquinela (2017) retoman los aportes de los ESCT sobre el proceso de construcción del conocimiento científico para aproximarse a la relación que los psicólogos vinculados a políticas sociales en Uruguay establecen con la población.

El papel concedido por la TAR a las entidades no humanas como agentes activos dentro de la red también ha tenido eco en los estudios sobre las políticas públicas. En este grupo se ubican algunos trabajos (Hoyler y Campos 2019; Leite 2018; S. Martínez 2021; Rivera-Aguilera 2017) que, acogiendo el llamado de Hull (2012) con respecto a la necesidad de analizar los documentos como objetos etnográficos en su propio derecho y no solamente como textos a ser interpretados, reconocen el papel de los documentos de política pública como mediadores que transforman y traducen el significado o los elementos que ellos transportan. En una dirección similar, los estudios de Mora (2016), E. Martínez (2016), Soto et al. (2017) y Ramos (2016; 2018) analizan las funciones performativas de los instrumentos de registro, gestión, medición y evaluación de políticas públicas, los dos primeros en Colombia y los tres últimos en Chile.

Por último, es preciso señalar que un importante grupo de investigaciones integra ambas perspectivas de análisis - la socioantropológica y la sociología de la traducción- al considerar las políticas públicas como ensamblajes de diversas racionalidades y técnicas de gobierno dirigidas a la administración de las poblaciones. En este grupo se sitúan algunos trabajos sobre las políticas urbanas en dos ciudades colombianas (Pérez 2010), el dispositivo de control migratorio en Brasil (Jardim 2016), las políticas de salud en Chile (Castillo et al. 2017), el Programa Prospera en México (Ortiz 2017), el sistema de evaluación del sistema educativo en Chile (Ramos 2018) y las políticas anticorrupción en Colombia (Arenas y Alzate 2019). 


\section{A manera de conclusión}

En este artículo hemos presentado los principales aportes de la antropología y de la sociología de la traducción al estudio de las políticas públicas que, al hacer de ellas un objeto de problematización, dejan atrás aquellas visiones instrumentalistas que daban por descontada su naturaleza y comportamiento. Ambos enfoques toman en consideración el nutrido elenco de actores que intervienen en el devenir de estas políticas, los intereses y valores que animan sus decisiones, los programas de acción que estos ponen en marcha para encauzar la conducta de otros y las diversas estrategias que despliegan para conseguir la adhesión de sus adversarios.

Esta manera de aproximarse al fenómeno conduce al reconocimiento de las políticas públicas como arenas de lucha en las que múltiples actores, con posiciones diferenciadas en el espacio social, libran contiendas en torno al ejercicio del poder y la dominación. Dichas perspectivas también comparten un abordaje distintivo del llamado "éxito" o "fracaso" de las políticas, en el que estos aspectos no son el resultado del grado de adecuación entre los modelos y las condiciones reales de implementación, sino más bien el producto temporal de asociaciones inestables.

En lo que tiene que ver con las contribuciones de los enfoques socioantropológicos, vimos también cómo estas investigaciones han aportado a la comprensión de, al menos, tres fenómenos: 1) los efectos producidos por las políticas sobre las poblaciones asistidas, principalmente en lo que tiene que ver con los procesos de subjetivación que de allí se derivan; 2) la intrincada relación entre las burocracias y los destinatarios de las políticas, mediante el análisis de las prácticas y representaciones que se ponen en juego en los encuentros entre unos y otros, así como de las respuestas diversas de los ciudadanos a los procesos de intervención; 3) el papel desempeñado por las burocracias en la formulación y ejecución de las políticas.

A partir de la revisión efectuada, pudimos constatar que, a excepción de los estudios enfocados en los saberes expertos, gran parte de la literatura antropológica se concentra en las interacciones entre las llamadas burocracias del nivel callejero (Lipsky [1980] 1999) y las poblaciones beneficiarias, mientras que los arquitectos de las políticas, generalmente situados en rangos intermedios o superiores de las jerarquías burocráticas, han sido mucho menos atendidos. Este vacío en la literatura plantea una prometedora veta de análisis en términos del conocimiento de los sistemas de valores y principios que informan las decisiones de los responsables de la formulación y evaluación de las políticas públicas.

Con respecto a la aplicación del enfoque de la sociología de la traducción al análisis de las políticas públicas, encontramos tres grandes grupos de trabajos: 
1) aquellos que asumen esta perspectiva de manera integral al adoptar una mirada de las políticas como un ensamblaje de entidades heterogéneas (actores humanos y no-humanos, colectivos e individuales, recursos materiales y simbólicos, etc.) articuladas temporalmente alrededor de su proceso de construcción; 2) aquellos que se apoyan en el uso de algunas categorías pertenecientes a este marco analítico para el estudio de políticas y programas particulares; 3) los que se ocupan específicamente de la mediación ejercida por ciertos dispositivos de inscripción. Al integrar en sus análisis la larga cadena de asociaciones, traducciones y desplazamientos de significados que redefinen permanentemente el entramado de las políticas públicas, estas investigaciones rompen con la representación lineal de las políticas como un proceso escalonado que avanza de manera progresiva entre sus diferentes etapas. A pesar del reconocimiento que sus autores hacen de la capacidad de agencia de los actantes no humanos, los estudios dedicados a los procesos de construcción de los documentos de política siguen siendo escasos.

\section{Referencias}

Agudo, Alejandro. 2009. “Conocimiento, lenguaje, poder e intermediación. Perspectivas contemporáneas en la antropología de las políticas públicas”. Estudios Sociológicos 27 (79): 63-110. https://doi.org/10.24201/ES.2009V27N79.263

—. 2011. "Mejoras privadas, beneficios colectivos: la producción y subversión de regímenes globales de política social en Chiapas”. En (Trans)formaciones del estado en los márgenes de Latinoamérica. Imaginarios alternativos, aparatos inacabados y espacios transnacionales, editado por Alejandro Agudo y Marco Estrada, 231-283. Ciudad de México: Universidad Iberoamericana; Colegio de México.

Amantino, Jackeline y José Arimatéia. 2017. "Análise da instrumentação da ação pública a partir da teoría do ator-rede: tecnología social e a educação no campo en Rondônia”. Revista de Administração Pública 51 (3): 407-430. https://doi.org/10.1590/0034-7612153318

Aparicio, Ricardo. 2005. "Intervenciones etnográficas a propósito del sujeto desplazado: estrategias para (des)movilizar una política de la representación”. Revista Colombiana de Antropología 41: 135-169. https://doi.org/10.22380/2539472X.1205

Apthorpe, Raymond. 1997. "Writing development policy and policy analysis plain or clear: on language, genre and power". En The anthropology of policy: critical perspectives on governance and power, editado por Cris Shore y Susan Wright, 43-58. Nueva York: Routledge.

Arenas Hernández, Sandra Lucía y Velmar David Alzate Pérez. 2019. "Políticas públicas como tecnologías de gubernamentalidad: una propuesta investigativa a partir del estudio de la lucha anticorrupción”. Ópera 25: 55-74. https://doi.org/10.18601/16578651.n25.04 
Assusa, Gonzalo. 2014. "Dispuestos a trabajar. Aproximación a la visión de los agentes estatales de un programa de empleo para jóvenes en Argentina”. Revista de Antropología Social 23: 181-208. https://doi.org/10.5209/rev_RASO.2014.v23.46730

Auyero, Javier. 2013. Pacientes del estado. Buenos Aires: Eudeba.

Barbato Bevilaqua, Ciméa. 2005. "Entre o previsível e o contingente: etnografía do proceso de decisão sobre uma política de ação afirmativa”. Revista de Antropologia 48 (1): 167-225. https://doi.org/10.1590/S0034-77012005000100005

Buchely, Lina. 2015. Activismo burocrático. La construcción cotidiana del principio de legalidad. Bogotá: Ediciones Uniandes.

Cabrera, Juan. 2011. "Pensar e intervenir el territorio a través de la teoría del actor-red". Athenea Digital 11 (1): 217-223. https://doi.org/10.5565/rev/athenead/v11n1.831

Callon, Michel. 1995. "Algunos elementos para una sociología de la traducción: la domesticación de las vieiras y los pescadores de la bahía de St. Brieuc”. En Sociología de la ciencia y la tecnología, compilado por Manuel Iranzo, Rubén Blanco, Teresa González, Cristóbal Torres y Alberto Cotillo, 259-282. Madrid: Consejo Superior de Investigaciones Científicas.

-. 1998. "El proceso de construcción de la sociedad. El estudio de la tecnología como herramienta para el análisis sociológico". En Sociología simétrica. Ensayos sobre ciencia, tecnología y sociedad, compilado por Miquel Domenech y Francisco Tirado, 143-170. Barcelona: Gedisa Editorial.

Castillo, Jorge, Marjorie Espejo, Jorge Tapia, Miguel Catalán, José Toro y Mariana Gálvez. 2017. "Tecnologías, episteme y subjetivación en un régimen de garantías en salud”. Psicoperspectivas 16 (3): 6-16. https://doi.org/10.5027/psicoperspectivas-vol16-issue3-fulltext-1069

Castillo, Jorge, María Winkler y Héctor Berroeta. 2019. "Del empoderamiento a la prehensión: un análisis de programas sociales desde la teoría del actor-red”. Athenea Digital 19 (3): e1833. https://doi.org/10.5565/rev/athenea.1833

Chao, Daniel. 2019. "Especial y marginal: hacia una problematización de la intervención estatal en la salud de los veteranos de Malvinas (1984-2000)”. Salud Colectiva 15: e2205. https://doi.org/10.18294/sc.2019.2205

Chávez Bidart, Jorge, Cecilia Hontou y Pablo Piquinela. 2016. "La proximidad en las políticas públicas: tensiones entre el abordaje de la vulnerabilidad social y la evidencia científica”. Rev. Polis e Psique 6 (2): 5-24. https://doi.org/10.22456/2238-152X.65724

Chávez Bidart, Jorge y Pablo Piquinela. 2017. "Expertos en ciencia, legos en política: ¿qué psicólogos para las políticas públicas?”. Psicoperspectivas 16 (2): 88-99. https://www.psicoperspectivas.cl/index.php/psicoperspectivas/article/view/1006/843

Chávez Bidart, Jorge y Leonardo Riet. 2015. "Equipos técnicos en acción. Estudio de políticas públicas desde la teoría del actor red”. En Psicologia, tecnologia e sociedade. Controvérsias metodológicas e conceituaies para uma análise das práticas de subjetivação, editado por Arthur Arruda, Adriana Molas y Jimena Carrasco, 97-116. Río de Janeiro: Nau Editora.

Corrêa, Jacy y Mary Jane Spink. 2014. "Multiplicidade de 'pobrezas' nas políticas públicas contemporáneas: contribuições do construccionismo social e da teoria ator-rede (TAR)". Dialogo Canoas 27: 9-23. https://revistas.unilasalle.edu.br/index.php/Dialogo/article/ view/1815 
Da Silva, Mónica y Laura López. 2014. "Pensando el 'Plan Ceibal' desde la perspectiva de la acción pública y la teoría del actor-red”. Athenea Digital 14 (1): 49-68. https://doi. org/10.5565/rev/athenead/v14n1.939

D’Alessandro-Nogueira, Renzo y Alma González-Cabañas. 2014. "Siete controversias capitales: análisis de la implementación del Programa Maíz Solidario en Los Altos de Chiapas”. Revista LiminaR. Estudios Sociales y Humanísticos 12 (2): 129-147. https://go.gale.com/ps/i. do?id=GALE\%7CA392478920\&sid=googleScholar\&v=2.1\&it=r\&linkaccess=abs\&issn=16658 027\&p=IFME\&sw=w\&userGroupName=anon\%7E85b4664d

Dallorso, Nicolás Santiago. 2012. "Notas sobre el uso del concepto de dispositivo para el análisis de programas sociales”. Espiral, Estudios sobre Estado y Sociedad 19 (54): 54-74. http:// www.scielo.org.mx/scielo.php?script=sci_arttext\&pid=S1665-05652012000200002

Dapuez, Andrés. 2016. "Development and the temporality of its exchange. How an Eastern Yucatec village made cash transfer promises accountable”. Antípoda 26: 159-177. https:// doi.org/10.7440/antipoda26.2016.07

Dávila, Juana. 2017. "A land of lawyers, experts and 'men without land': the politics of land restitution and the techno-legal production of 'dispossessed people' in Colombia”. Tesis doctoral, Department of Anthropology, Harvard University, Cambridge.

Dean, Mitchell. 2002. "Liberal government and authoritarianism”. Economy and Society 31 (1): 37-61. https://doi.org/10.1080/03085140120109240

Diehl, Eliana y Jean Langdon. 2015. "Transformações na atenção à saúde indígena: tensões em um contexto indígena brasileiro”. Universitas Humanística 80: 213-236. https://doi. org/10.11144/Javeriana.UH80.tasi

Escobar, Arturo. (1995) 2007. La invención del tercer mundo. Construcción y deconstrucción del desarrollo. Caracas: Fundación Editorial El Perro y la Rana.

Fagundez, Daniel. 2018. "Análisis de las territorialidades urbanas en los diseños de intervención para el abordaje de adolescentes en situación de calle”. Psicoperspectivas 17 (2). https://doi.org/10.5027/psicoperspectivas-vol17-issue2-fulltext-1146

Fagundez, Daniel y Diego González. 2018. "Definir para gobernar: políticas dirigidas a niños, niñas y adolescentes en situación de calle en Uruguay”. Revista Latinoamericana de Ciencias Sociales, Niñez y Juventud 16 (2): 995-1008. https://doi.org/10.11600/1692715x.16223

Faustino, Graciele Oliveira y Simone Maria Hüning. 2015. "El Plano Juventude Viva y la red de políticas de juventud para enfrentar la violencia en Alagoas”. Estudos e Pesquisas em Psicologia 15 (4): 1413-1432. http://pepsic.bvsalud.org/scielo.php?script=sci_abstract \&pid=S1808-42812015000400017\&lng=en\&nrm=iso\&tlng=es

Ferguson, James. 1994. The anti-politics machine: “development”, depoliticization, and bureaucratic power in Lesotho. Minneapolis: University of Minnesota Press.

Foucault, Michel. 2006. Seguridad, territorio, población. Buenos Aires: Fondo de Cultura Económica.

Franco, Angélica. 2016. "Fronteras simbólicas entre expertos y víctimas de la guerra en Colombia”. Antípoda 24: 35-53. https://doi.org/10.7440/antipoda24.2016.03 
García, Sabina. 2016. "El multiculturalismo como modelo de gobernanza en Chile: estado, academia y brokers”. Universitas Humanística 82: 307-334. https://doi.org/10.11144/Javeriana.uh82.mmgc

Gracia Hincapié, Liliana. 2015. "Para uma antropologia da política pública: dinámicas da construção de políticas públicas para comunidades afrocolombianas”. Revista de Políticas Públicas da Universidade Federal do Maranhão 19 (1): 1-24. http://dx.doi.org/10.18764/21782865.v19n1p157-171

Grau, Marc, Lupicinio Íñiguez y Joseph Subirats. 2010. "La perspectiva sociotécnica en el análisis de políticas públicas”. Psicología Política 41: 61-80. https://portalrecerca. uab.cat/en/publications/la-perspectiva-sociot\%C3\%A9cnica-en-el-an\%C3\%A1lisis-depol\%C3\%ADticas-p\%C3\%BAblicas

Greenhalgh, Susan. 2008. Just one child: science and policy in Deng's China. Berkeley: University of California Press.

Grinberg, Julieta. 2008. "Transformaciones en el tratamiento de la niñez en riesgo. Reflexiones sobre un dispositivo de protección a la infancia en la ciudad de Buenos Aires”. Cuadernos de Antropología Social 27: 155-174. https://doi.org/10.34096/cas.i27.4334

Gutiérrez, Alicia y Gonzalo Assusa. 2015. "La articulación de lógicas laborales y lógicas domésticas en una política de empleo para jóvenes en Argentina. Individuo, autonomía y vínculos personales en el mundo del trabajo”. Antípoda 22: 21-44. https://doi.org/10.7440/ antipoda22.2015.02

Herron, James. 2003. "Animating the state: discourses of authority and intimacy in the Colombian Agrarian Bank”. Tesis doctoral, Department of Anthropology, University of Michigan, Ann Arbor.

Hornes, Martín Eduardo. 2014. "Transferencias condicionadas y sentidos plurales: el dinero estatal en la economía de los hogares argentinos”. Antípoda 18: 61-83. https://doi.org/ 10.7440/antipoda18.2014.04

-. 2020. "Expertos que diseñan el dinero: el caso de los programas de transferencias monetarias”. Estudios Sociológicos 38 (112): 141-171. https://doi.org/10.24201/es.2020v38n112.1756

Hornes, Martín Eduardo y Agustín Salerno. 2019. “Interpretaciones sobre la cercanía estatal en las áreas socio-asistenciales de dos municipios de la región metropolitana de Buenos Aires. Sentidos, relaciones y prácticas de los funcionarios políticos, los burócratas de la calle y los sectores asistidos entre 2007 y 2015”. Estudios Sociales del Estado 5 (10): 60-91. https://doi.org/10.35305/ese.v5i10.190

Hoyler, Telma y Pedro Campos. 2019. "A vida política dos documentos: notas sobre burocratas, políticas e papéis”. Revista de Sociologia e Política 27 (69): e.006. https://doi. org/10.1590/1678987319276906

Hull, Mathew. 2012. Government of paper. The materiality of bureaucracy in urban Pakistan. Berkeley: University of California Press.

Isacovich, Paula. 2013. "Hacer el estado. Regular la vida. Una etnografía de políticas de juventud”. Avá. Revista de Antropología 22: 33-56. http://www.ava.unam.edu.ar/images/22/ pdf/n22a02.pdf 
Jabs, Talita. 2013. "Dinheiro e moralidade no Bolsa Família: uma perspectiva etnográfica”. Tesis de Maestría en Antropología, Instituto de Filosofia e Ciências Humanas, Universidade Federale do Rio Grande do Sul, Porto Alegre.

Jaramillo, Alejandra. 2015. "Reshaping policy: creativity and everyday practices in an arts organisation in Mexico City”. Tesis doctoral, Sociology Department, University of York, York.

Jaramillo Marin, Jefferson. 2006. “Reubicación y restablecimiento en la ciudad. Estudio de caso con población en situación de desplazamiento”. Universitas Humanística 62: 143-168. https://revistas.javeriana.edu.co/index.php/univhumanistica/article/view/2213

-. 2008. "Representaciones y prácticas discursivas sobre la política de atención a la población en situación de desplazamiento. Estudio de caso en Bogotá”. Universitas Humanística 65: 197-236. http://www.scielo.org.co/scielo.php?pid=S0120-48072008000100010\&script= sci_abstract\&tlng=es

Jardim, Denise. 2016. "Imigrantes ou refugiados? As tecnologias de governamentalidade e o êxodo palestino rumo ao Brasil no século XX”. Horizontes Antropológicos 22 (46): 243-271. https://doi.org/10.1590/S0104-71832016000200009

Kerr Pontes, Alexandre, Ana Paula Santos Meza y Pedro Paulo Gastalho de Bicalho. 2015. "Ciência e política das drogas: as controversias em torno das políticas públicas de internação compulsória”. Estudos e Pesquisas em Psicologia 15 (4): 1433-1450. https://doi. org/10.12957/epp.2015.20280

Latour, Bruno. 1992. Ciencia en acción. Cómo seguir a los científicos e ingenieros a través de la sociedad. Barcelona: Editorial Labor.

-. 2008. Reensamblar lo social. Una introducción a la teoría del actor-red. Buenos Aires: Manantial.

Law, John. 1998. "Del poder y sus tácticas. Un enfoque desde la sociología de la ciencia”. En Sociología simétrica. Ensayos sobre ciencia, tecnología y sociedad, compilado por Miquel Domenech y Francisco Tirado, 63-107. Barcelona: Gedisa Editorial.

Leite, Lilian. 2018. "Dos eventos documentados aos documentos manejados: a política de saúde mental brasileira em disputa”. Anuário Antropológico 43 (2): 261-284. https://doi. org/10.4000/aa.3282

Li, Tania. 2007. The will to improve. Governmentality, development and the practice of politics. Durham; London: Duke University Press.

Lipsky, Michael. (1980) 1999. "La burocracia en el nivel callejero: la función crítica de los burócratas en el nivel callejero”. En Clásicos de la administración pública, editado por Jay Shafritz y Albert Hyde, 780-794. Ciudad de México: Fondo de Cultura Económica.

Llanes, Carolina. 2009. "Desafíos y adaptaciones en el liderazgo femenino sociopolítico. Programa Familias en Acción en Maicao-La Guajira, Colombia”. Estudios Sociológicos 27 (81): 909-932. https://doi.org/10.24201/ES.2009V27N81.329

Long, Norman. (2001) 2007. Sociología del desarrollo: una perspectiva centrada en el actor. Ciudad de México: El Colegio de San Luis. 
Lozano, Juan Ignacio. 2016. “Los movimientos sociales en el 'retorno' del Estado. Experiencias de participación en la gestión estatal de la provincia de Buenos Aires, Argentina”. Universitas Humanística 82: 163-187. https://doi.org/10.11144/Javeriana.uh82.msre

Mancini, Inés. 2016. "Alcanzar la inclusión social o rescatarse, dos lógicas para la transformación. Análisis de una política de prevención social del delito y el cambio de sus beneficiarios”. Estudios Sociales del Estado 2 (4): 181-209. https://doi.org/10.35305/ese.v2i4.95

Mariano, Silvana Aparecida. 2013. "Estrategias de las usuarias de la asistencia social: una lectura en torno a las relaciones de poder”. Estudios Sociológicos 31 (91): 141-166. https:// estudiossociologicos.colmex.mx/index.php/es/article/view/121/121

Martínez, Eddier. 2016. "El Sivigila, una infraestructura que moviliza enfermedades, prácticas y políticas de vigilancia en salud pública”. Revista Colombiana de Sociología 39 (2): 283-302. https://doi.org/10.15446/rcs.v39n2.58977

Martínez Ramírez, Fermín. 2019. "Experiencia laboral en el programa Argentina Trabaja. Significaciones de los trabajadores en torno al ingreso y permanencia en cooperativas de Berisso, Ensenada y La Plata”. Estudios Sociales del Estado 5 (10): 92-127. https://doi. org/10.35305/ese.v5i10.191

Martínez B., Sandra. 2017. Encuentros con el estado: burocracias y colonos en la frontera amazónica (1960-1980). Cali: Programa Editorial Universidad del Valle.

- 2019. "Entre bastidores: etnografía de la política pública para los afrodescendientes en Cali, Colombia”. Revista Colombiana de Antropología 55 (1): 213-238. https://doi. org/10.22380/2539472X.577

-. 2021. "Este proceso ha sido de piel, no ha sido de escritorio': itinerario del documento de la política pública CaliAfro”. Revista de Antropología Social 30 (1): 69-82. https://doi. org/10.5209/raso.74620

Medan, Marina. 2018. Prevención social y delito juvenil. El gobierno de la juventud en riesgo en el AMBA: entre la seguridad y la inclusión. Buenos Aires: Universidad de Buenos Aires, Teseo.

Mora-Gámez, Fredy. 2016. "Reconocimiento de víctimas del conflicto armado en Colombia: sobre tecnologías de representación y configuraciones de Estado”. Universitas Humanística 82: 75-101. https://doi.org/10.11144/Javeriana.uh82.rvca

Mosse, David. 2005. Cultivating development. An ethnography of aid policy and practice. Londres; Arbor: Pluto Press.

Nuijten, Monique. 2003. Power, community, and the State. The political anthropology of organisation in México. Londres: Pluto Press.

Ortiz, Samantha. 2017. "Gubernamentalidad y política pública: estudio alternativo del programa Prospera”. Revista Mexicana de Sociología 79 (3): 543-570. http://dx.doi.org/10.22201/ iis.01882503p.2017.3.57677

Osorio, Abraham. 2014. “¿Acción y malestar? Las tácticas de resistencia de los menores internados en las instituciones asistenciales”. Estudios Sociológicos 32 (94): 103-129. https:// estudiossociologicos.colmex.mx/index.php/es/article/view/31 
Osorio, Ana. 2011. "Emergencia de nuevas formas de subjetividad política como objeto de políticas públicas sociales. La propiedad horizontal en el Sistema Distrital de Participación Ciudadana”. Tesis de maestría, Facultad de Ciencias Políticas y Relaciones Internacionales, Pontifica Universidad Javeriana, Bogotá.

Perelmiter, Luisina. 2016. Burocracia plebeya. La trastienda de la asistencia social en el Estado argentino. San Martín: Universidad Nacional de Gral. San Martín, Unsam Edita.

Pérez, Federico. 2010. "Laboratorios de reconstrucción urbana: hacia una antropología de la política urbana en Colombia”. Antípoda 10: 51-84. https://dx.doi.org/10.7440/antipoda10.2010.04

Ramos-Zincke, Claudio. 2016. La producción de la pobreza como objeto de gobierno. Santiago de Chile: Ediciones Universidad Alberto Hurtado.

—. 2018. "Dispositivo de evaluación y gubernamentalidad del sistema educacional: entretejimiento de ciencia social y poder”. Cinta Moebio 61: 41-55. https://dx.doi.org/10.4067/S0717$554 X 2018000100041$

Rap, Edwin. 2008. "Produciendo el éxito en la política pública. La transferencia del manejo del riego en México”. Estudios Sociológicos 26 (77): 249-285. https://dialnet.unirioja.es/descarga/articulo/6164611.pdf

Recalde, Gabriela. 2019. “'En lo que esté a mi alcance les ayudo’. Los funcionarios de base y las víctimas en el proceso de declaración para la inscripción en el Registro Único de Víctimas del conflicto armado". En Etnografías burocráticas. Una nueva mirada a la construcción del Estado en Colombia, compilado por Isabel Jaramillo y Lina Buchely, 173-216. Bogotá: Universidad de los Andes.

Restrepo, Ana. 2010. "Las políticas públicas como mecanismos de reproducción del Estado: una mirada desde la Política Pública de Juventud de Bogotá”. Antípoda 10: 85-106. https:// dx.doi.org/10.7440/antipoda10.2010.05

Rivera-Aguilera, Guillermo. 2017. "Los procesos de influencia global/local en políticas públicas: una propuesta metodológica”. Psicoperspectivas 16 (3): 110-120. https://dx.doi. org/10.5027/psicoperspectivas-vol16-issue3-fulltext-1092

Rose, Nikolas. 1996. "Governing 'advanced' liberal democracies. En Foucault and political reason: liberalism, neo-liberalism and rationalities of government, editado por Andrew Barry, Thomas Osborne y Nikolas Rose, 37-64. Chicago; Londres: University of Chicago Press.

Rose, Nikolas y Peter Miller. 1992. "Political power beyond the state: problematics of government”. The British Journal of Sociology 43 (2): 173-205. https://doi.org/10.2307/591464

Salaini, Cristian y Denise Jardim. 2015. "Batalha dos papéis: notas sobre as tensões entre procedimentos escritos e memória na regularização fundiária de terras de quilombos no Brasil”. Universitas Humanística 80: 189-212. https://doi.org/10.11144/Javeriana.UH80. bdpn

Saldi, Leticia. 2016. "El Estado-sistema frente a la cuestión indígena. Análisis de los modos de reconocimiento de derechos a la identidad huarpe, al agua y a las tierras comunales en Mendoza (Argentina)”. Universitas Humanística 82: 191-218. https://doi.org/10.11144/Javeriana.uh82.esfc 
Salej, Silvio, Ana Ardila y Josiane Bragato. 2016. "Autonomía integrada y el poder discrecional de las burocracias: evidencias de un programa de riesgo geológico en Belo Horizonte, Brasil”. Estudios Sociológicos 34 (101): 353-381. http://estudiossociologicos.colmex.mx/index.php/es/article/view/1452/1467

Salete, Irme, Celso Tondin, Misael Szytko, Eduarda Moro, Tiago Ramos y Giovane Biazi. 2015. "Circulação de crianças e adolescentes na rede tecida pelo Programa de Erradicação do Trabalho Infantil (PETI)”. Estudos e Pesquiss em Psicologia 15 (4): 1345-1362. https://doi. org/10.12957/epp.2015.20275

Schöngut-Grollmus, Nicolás. 2017. "Ensamblajes socio-técnicos para la producción de intervenciones psicosociales en un programa de Servicio Nacional de Menores en Chile”. Psicoperspectivas 16 (3): 40-51. https://doi.org/10.5027/psicoperspectivas-vol16-issue3-fulltext-1049

Scott, James. 1998. Seeing like state. How certain schemes to improve human condition have failed. New Haven; Londres: Yale University Press.

Shore, Cris. 2010. "La antropología y el estudio de la política pública: reflexiones sobre la 'formulación’ de las políticas”. Antípoda 10: 21-49. https://doi.org/10.7440/antipoda10.2010.03

Shore, Cris y Susan Wright. 1997. "Policy: a new field of anthropology”. En The anthropology of policy: critical perspectives on governance and power, editado por Cris Shore y Susan Wright, 3-39. Nueva York: Routledge.

-. 2011. "Conceptualising policy: technologies of governance and the politics of visibility". En Policy worlds. Anthropology and the analysis of contemporary power, editado por Cris Shore, Susan Wright y Davide Però, 1-25. Nueva York; Oxford: Berghahn Books.

Soto, Álvaro, Carla Fardella, Alan Valenzuela y Francisca Carvajal. 2017. "Las funciones performativas de los instrumentos en las redes de acción pública”. Psicoperspectivas 16 (3): 76-86. https://doi.org/10.5027/psicoperspectivas-vol16-issue3-fulltext-999

Ureta, Sebastián. 2014. "Policy assemblages: proposing an alternative conceptual framework to study public action”. Policy Studies 14 (3): 303-318. https://doi.org/10.1080/014 42872.2013.875150

-. 2015. Transantiago, human devices, and the dream of a world-class society. Massachusetts: The MIT Press.

Wanderley, Fernanda. 2009. "Prácticas estatales y el ejercicio de la ciudadanía: encuentros de la población con la burocracia en Bolivia”. Íconos 34: 67-79. https://doi.org/10.17141/iconos.34.2009.334

Wedel, Janine, Cris Shore, Gregory Feldman y Stacy Lathrop. 2005. “Towards an anthropology of public policy”. The Annals of the American Academy of Political and Social Sciences 600: 30-51. https://doi.org/10.1177/0002716205276734 